\title{
A Proof of the Binomial Theorem, with some Applications.
}

By Professor Gibson.

(Read and Received 12th December 1919.)

Many proofs of the Binomial Theorem have been given, and the proof which I give in this note has not the slightest claim to be considered new. My only object in giving it is to call attention to the fact that it depends merely on the rule of integration by parts, and provides a form of the remainder that in many applications is much simpler than any of the forms associated with Taylor's Theorem. Of its usefulness in this latter respect I give an example from the asymptotic expression for a Bessel function.

If $t$ is a real variable, and if $1+x t$ is not zero for the range $0 \leqq t \leqq 1$ then

so that

$$
\int_{0}^{1}(1+x t)^{n-1} d t=\frac{(1+x)^{n}-1}{n x}
$$

$$
(1+x)^{n}=1+n x \int_{0}^{1}(1+x t)^{n-1} d t
$$

Here $x$ and $n$ may be complex, with the usual restriction that when $n$ is not integral the value of $(1+x)^{n}$ when $x$ is zero is unity.

Now integrate by parts, but take

$$
\int 1 \cdot d t=-(1-t), \int(1-t)^{r} d t=-(1-t)^{r+1} /(r+1)
$$

thus

$$
\begin{aligned}
\int_{0}^{1}(1+x t)^{n-1} d t & =\left[-(1-t)(1+x t)^{n-1}\right]_{0}^{1} \\
& +(n-1) x \int_{0}^{1}(1-t)(1+x t)^{n-2} d t \\
& =1+\frac{n-1}{2} x \int_{0}^{1} 2(1-t)(1+x t)^{n-2} d t .
\end{aligned}
$$

Equation (1) now becomes

$$
(1+x)^{n}=1+n x+\frac{n(n-1)}{1.2} x^{2} \int_{0}^{1} 2(1-t)(1+x t)^{n-2} d t \ldots \ldots
$$


Another integration gives for $(1+x)^{n}$ the expression

$$
1+n x+\frac{n(n-1)}{1.2} x^{2}+\frac{n(n-1)(n-2)}{1.2 .3} \int_{0}^{1} 3(1-t)^{2}(1+x t)^{n-3} d t \ldots \ldots .
$$

Assume now the general form

$$
(1+x)^{n}=1+{ }_{n} C_{1} x+{ }_{n} C_{2} x^{2}+\ldots+{ }_{n} C_{r-1} x^{r-1}+R \ldots \ldots \ldots \ldots
$$

where

$$
R={ }_{n} C_{r} x^{r} \int_{0}^{1} r(1-t)^{r-1}(1+x t)^{n-r} d t .
$$

Of course ${ }_{n} C_{r}=\frac{n(n-1) \ldots(n-r+1)}{r !}$; it makes the expressior for $R$ more symmetrical to write $r(1-t)^{r-1}$, thus making the coefficient the complete ${ }_{n} C_{r}$.

By integration by parts it is readily found that

$$
R={ }_{n} C_{r} x^{r}+{ }_{n} C_{r+1} x^{r+1} \int_{0}^{1}(r+1)(1-t)^{r}(1+x t)^{n-r-1} d t
$$

so that the form (4) holds for every $r$.

When $x$ and $n$ are real it is easy to express $R$ in the usual forms. For example, $(1+x t)^{n-r}$ lies between 1 and $(1+x)^{n-r}$, so that $R$ lies between ${ }_{n} C_{r} x^{r}$ and ${ }_{n} C_{r} x^{r}(1+x)^{n-r}$, and is therefore of the form ${ }_{n} C_{r} x^{r}(1+\theta x)^{n-r}$ where $0<\theta<1$. But the expression for $R$ as an integral is specially useful when $x$ and $n$ are complex; in such cases the usual form for the remainder is not directly applicable.

As an illustration I take the expression for $G_{n}(z)$ on p. 272 of Dr MacRobert's Functions of a Complex Variable. There an expression for the remainder in

$$
\left(1+\frac{i \zeta}{2 z}\right)^{n-\frac{1}{2}}
$$

is required. In this case $n, z$ and $\zeta$ are complex, the amplitude of $\zeta$ lying between $-\pi / 2$ and $\pi / 2$ and that of $\phi$ between $-\pi / 2$ and $3 \pi / 2$. The investigation would be considerably simplified if $\zeta$ were real and positive.

Now let $z=\rho e^{i \phi}$; then

$$
\frac{i \zeta}{2 z}=\frac{\zeta}{2 \rho}(i \cos \phi+\sin \phi),-\frac{\pi}{2}<\phi<\frac{3 \pi}{2} \text {. }
$$

If $\zeta$ is resl and positive, this expression is not real and negative unless $\phi$ is $-\pi / 2$ or $3 \pi / 2$ and these values of $\phi$ are excluded. Hence $1+i \zeta t / 2 z$ can not vanish, $t$ being real, unless $z$ is on the negative part of the imaginary axis. The form (5) for the 
remainder can therefore be used when $x=i \zeta / 2 z$. When $n-\frac{1}{2}$ is put for $n$ in (5) we get

$$
R={ }_{n-1} C_{r}\left(\frac{i \zeta}{2 z}\right)^{r} \int_{0}^{1} r(1-t)^{r-1}\left(1+\frac{i \zeta t}{2 z}\right)^{n-t-r} d t .
$$

Let $1+\frac{i \zeta t}{2 z}=a e^{i \psi}$; then it is easy to see that

$$
a \geqq 1 \text { if } 0 \leqq \phi \leqq \pi,
$$

but $a \geqq|\cos \phi|$ if $-\frac{\pi}{2}<\phi<0$ or $\pi<\phi<\frac{3 \pi}{2}$ while

$-\pi<\psi<\pi$.

If $n=\alpha+i \beta$ and $r>\alpha-\frac{1}{2}$ we now find, $a_{0}$ being 1 or $|\cos \phi|$,

$$
\left|\left(1+\frac{i \zeta t}{2 z}\right)^{n-\frac{1}{2}-r}\right| \leqq \frac{e^{\pi \beta_{0}}}{a_{0}^{r+\frac{1}{2}-\alpha}}, \beta_{0}=|\beta|,
$$

and therefore

$$
\begin{aligned}
|R| \leqq \mid{ }_{n-\frac{1}{2}} C_{r} & \left(\frac{i \zeta}{2 z}\right)^{r} \mid \frac{e^{\pi \beta_{0}}}{a_{0}^{r+\frac{1}{2}-\alpha}} \\
& =\left|\frac{\Gamma\left(n+\frac{1}{2}\right)}{\Gamma(r+1) \Gamma\left(n+\frac{1}{2}-r\right)}\right| \frac{e^{\pi \beta_{0}}}{a_{0} r+\frac{1}{2}-\alpha} \mid \cdot \frac{\zeta}{|2 z|^{r}} .
\end{aligned}
$$

The part of $G_{n}(z)$ for which this value of $R$ is required is, but for a factor which does not concern us,

$$
\frac{1}{\Gamma\left(n+\frac{1}{2}\right)} \int_{0}^{\infty} e^{-\zeta} \zeta^{n-1} R d \zeta,
$$

and therefore the modulus of this is less than

$$
\begin{gathered}
\left|\frac{1}{\Gamma(r+1) \Gamma\left(n+\frac{1}{2}-r\right)}\right| \frac{e^{\pi \beta_{0}}}{a_{0}^{r+\frac{1}{2}-a}} \cdot \frac{1}{|2 z|^{r}} \int_{0}^{\infty} e^{-\zeta} \zeta^{n+r-1} d \zeta \\
=\left|\frac{\Gamma\left(n+\frac{1}{2}+r\right)}{\Gamma(r+1) \Gamma\left(n+\frac{1}{2}-r\right)}\right| \frac{e^{\pi \beta_{0}}}{a_{0}^{r+\frac{1}{2}-a}} \frac{1}{|2 z|^{r}} .
\end{gathered}
$$

In many investigations which require a limit for the remainder in the Binomial Expansion it will be found, I think, that the form (5) is much simpler than any of those usually associated with Taylor's Theorem. In fact none of these forms is directly applicable when the variable is complex and their application demands tedious transformations. 
As is well known, we could obtain Maclaurin's expansion of $f(x)$ by the method of integration by parts, as has been done for the expansion of $(1+\dot{x} t)^{n}$. Thus if $f^{\prime}(u)$ denote $\frac{d f(u)}{d u}$ we have

$$
\int_{0}^{1} f^{\prime}(x t) d t=\frac{1}{x}\{f(x)-f(0)\} \text { or } f(x)=f(0)+x \int_{0}^{\mathrm{t}} f^{\prime}(x t) d t .
$$

Again

$$
\int_{0}^{1} f^{\prime}(x t) d t=\left[-(1-t) f^{\prime}(x t)\right]_{0}^{1}+x \int_{0}^{1}(1-t) f^{\prime \prime}(x t) d t
$$

so that

$$
f(x)=f(0)+x f^{\prime}(0)+\frac{x^{2}}{1.2} \int_{0}^{1} 2(1-t) f^{\prime \prime}(x t) d t
$$

and the remainder would be

$$
\frac{x^{n}}{n !} \int_{0}^{1} n(1-t)^{n-1} f^{(n)}(x t) d t
$$

